\title{
Assessment of Risk of Carrier Waves in the Assisted Reproductive Laboratory
}

\author{
Samuel D. Prien1,2, Jessica Smith², Christy Barron², Joseph Martin², Naghma Farooqi1, \\ Alita Loveless' ${ }^{1}$ Amy Van Gheem1', Lindsay L. Penrose1 \\ ${ }^{1}$ Department of Animal and Food Sciences, Texas Tech University, Lubbock, USA \\ ${ }^{2}$ Department of Obstetrics and Gynecology, Texas Tech University Health Sciences Center, \\ Lubbock, USA \\ Email: Samuel.prien@ttuhsc.edu
}

Received 17 July 2015; accepted 30 August 2015; published 2 September 2015

Copyright (C) 2015 by authors and Scientific Research Publishing Inc.

This work is licensed under the Creative Commons Attribution International License (CC BY). http://creativecommons.org/licenses/by/4.0/

(c) (i) Open Access

\begin{abstract}
Assisted reproductive technology (ART) laboratories represent the marriage of the most basic of biological activities with the most cutting edge technologies. While this association has worked well, the mixture of biology and technology can create risks to normal embryo development. Recently a significant amount of literature has explored the risks of manmade, electrically induced magnetic fields and carrier waves on reproduction, which some studies have suggested will lower functional gamete numbers in the males and potentially induce genetic issues in embryos. However, little is known about these phenomena within the ART laboratory, a laboratory filled with electronic equipment. The object of the present study was to explore the potential exposure of gametes and early stage embryos to two of the most prevalent fields and waves utilized in manmade technologies seen in the general environment, electromagnetic fields (EMF) and radio frequency waves (RF), and determine the effect varying levels of these energetic forces had on gamete function and embryo development. Results indicated that while extremely high concentrations of EMF (approximately 50-100X of laboratory background) caused negative outcomes in both gametes and embryos, levels consistent will the majority of lab equipment did not appear to impact growth, or function. Further, even extremely high RF appeared to have no impact cellular function. Results suggest few issues with EMF or RF on gamete and embryo function at normal laboratory levels for the relatively short exposure times seen in the ART laboratory.
\end{abstract}

\section{Keywords}

Assisted Reproductive Technologies, Electromagnetic Fields, Radio Frequency Waves, Embryos, Sperm

How to cite this paper: Prien, S.D., Smith, J., Barron, C., Martin, J., Farooqi, N., Loveless, A., Van Gheem, A. and Penrose, L.L. (2015) Assessment of Risk of Carrier Waves in the Assisted Reproductive Laboratory. Open Journal of Obstetrics and Gynecology, 5, 535-541. http://dx.doi.org/10.4236/ojog.2015.510077 


\section{Introduction}

Magnetic fields and energized carrier waves are naturally occurring phenomena seen throughout the universe. On Earth these forces are observed in varying scales from the planetary gravity which holds everything in the environment to the surface to more localized examples such as lightning and volcanic eruptions. These forces represent the background exposures unavoidable in everyday life. However, beginning with the first commercial power plant in the late eighteen hundreds and culminating with the explosion of wireless communication systems (i.e., microwave radios, Wi-Fi, cell phones and satellite communications) that have become so common in everyday life, exposure to higher intensity carrier waves and the accompanying ionizing radiation, at levels tens to hundreds of time that of background levels, has become almost equally unavoidable.

Of the various field and carrier waves generated by manmade technologies, electromagnetic waves (EMF) and radio frequency waves (RF) appear to be the most common. Numerous everyday electronic appliances produce EMF, while cellphones (and other wireless sources) and their associated support equipment are a major source of RF. Each represents a unique exposure with potentially disruptive effects on cellular function. Previous research has suggested high intensity EMF to be associated with various cancers, heart disease and neurological conditions [1] [2]. Similarly, RF, being a form of ionizing radiation, has been associated with various cancers [3] [4].

Recently, there has been significant concern about possible associations between devices which generate EMF and/or RF and reproduction. Both EMF and RF have been associated with lower sperm counts, motilities and gamete apoptosis [5]-[7]. Further, long-term exposures have been associated with arrested testicular development in fetuses and neonate [8]. Additionally, maternal exposures to EMF or RF have been related to poor pregnancy outcomes [9] [10].

Modern assisted reproductive technology (ART) laboratories are filled with equipment which produces both EMF and RF. However, most of the studies of the effect of EMF and RF on reproduction have focused on long-term exposures rather than the relatively short-term exposures associated with ART procedures. While previous studies have focus primarily on the effects of EMF and RF on reproductive organs and gametes in vivo, the present group of experiments were undertaken to determine the risks of EMF and RF for gametes and early stage embryos within the ART laboratory; focusing both on short-term exposure and the effects of field strength on cellular survival and function in vitro.

\section{Materials and Methods}

\subsection{Establishment of EMF Risks in an ART Laboratory}

The ART laboratory is full of equipment with the potential to generate electromagnetic fields. Prior to any experimentation, it was decided to measure EMF field strength throughout the laboratory using a magnetic field meter (F.W. Bell Model 4070 Gaussmeter; National Instrument Corp., Austin, TX). As EMF is known to decrease with distance from the electrical source, measurements were taken at locations where biological materials would be place in instrumentation as well as determining lowest and highest levels with the lab. Average background (Lowest level) was measured as $2 \mathrm{mG}$. Highest levels were located on the electric fan motors of the bio-hoods and exceeded $200 \mathrm{mG}$. Table 1 demonstrates levels measured across the lab, which were used for exposures levels in subsequent experiments.

\subsection{EMF and Sperm Cell Function}

A series of experiments were conducted to establish the effects of varying levels of EMF on sperm cell function. As the literature suggests little in terms of reproductive cell damage due to short-term exposure $(<3 \mathrm{hr})$ in a laboratory setting (average cell processing and holding time), it was determined that the first experiment should be to culture cells at the highest and lowest levels of EMF observed in the laboratory. In the initial experiments, porcine semen was obtained from six animals from the local research farm and extended using standard procedures in the extender Androhep (Mini-tube; Verona, WI). Two-15 mL samples were transferred to standard $110 \mathrm{~mL}$ culture plates (Falcon; Becton Dickinson; Franklin Lakes, NJ). One dish was placed directly on a slidewarmer ( $2 \mathrm{mG}$ EMF) at $37^{\circ} \mathrm{C}$ as a control. The second dish was exposed to a $>200 \mathrm{mG}$ field generated by placing the dish between two electromagnets for 20 minutes (approximate processing time for a human semen sample) and then transferred to the warmer for the duration of the trial. Aliquots of each sample were manually 
Table 1. Electromagnetic field strengths found in typical reproductive laboratory equipment.

\begin{tabular}{cc}
\hline Equipment & Field Strength \\
\hline Laboratory Background EMF & $2 \mathrm{mG}$ \\
Hood Work Surface & $8 \mathrm{mG}$ \\
Centrifuge & $36 \mathrm{mG}$ \\
Incubator & \\
Upper Shelf & $86 \mathrm{mG}$ \\
Middle Shelf & $32 \mathrm{mG}$ \\
Lower Shelf & $2 \mathrm{mG}$ \\
Highest Level Detected & \\
Hood Van Motor & $>200 \mathrm{mG}$ \\
\hline
\end{tabular}

observed for motility and forward progress at half-hour intervals between 0 - 3 hrs.

After the initial study demonstrated differences in cell function with EMF exposure, the process was repeated using the same procedure but with exposures equivalent to levels seen in standard laboratory equipment as seen in Table 1 (2, 30 and $90 \mathrm{mG}$ ). Samples were prepared as above and then exposed to the assigned field for 24 hrs with sampling done at $0,1,3,6,9,12$ and 24 hrs. The EMF fields were generated by placing the samples on the shelves of the incubator with the corresponding field strength and the incubator maintained the temperature $\left(37^{\circ} \mathrm{C}\right)$ and humidity $(95 \%)$, for the culture period.

Earlier studies on EMF and cellular function have suggested EMF might have a detrimental on cellular membranes. It would appear logical to expect an electrical field to disrupt the charge on the cellular membrane and therefore membrane permeability. To test this hypothesis, sperm samples from five boars were prepared and exposed to $>200 \mathrm{mG}$ EMF or a non-EMF control for 3 hrs. At times 0, 0.5, 1, 2, and 3 hrs, aliquots were taken for semen analysis using a computer assisted semen analyzer (CASA; IVOS, Hamliton Throne, Beverly, MA). An additional $0.5 \mathrm{~mL}$ sample the sperm was combined with 10 microns of fluorescein (Sigma-Aldrich: St. Louis, MO), a fluorescent dye. The cells were then observed at $1000 \mathrm{X}$ under a Lietz fluorescent microscope (Leica Microsystems; Buffalo Gobe, IL) with appropriate wavelength cubes, and classified as either permeabilized (fluorescing) or non-permeabilized, indicating the status of the cellular membrane.

Once studies with porcine semen had been completed, a small study was done with human semen to verify observed results. As in the previous animal study, human sperm samples were subjected to EMF fields of varying strengths for 24 hrs. Sperm samples from 5 paid donors were prepared for IUI using a standard sperm washing protocol. The final pellet was then resuspended in $4 \mathrm{~mL}$ of fresh Ham's F-10 (Irvine Scientific; Santa Ana, CA) and split into equal aliquots. The samples were subjected to three EMFs consistent with laboratory equipment (2, 30 and $90 \mathrm{mG}$ ) and an extremely high EMF of $>200 \mathrm{mG}$. At times 0, 1, 3 and $24 \mathrm{hrs}$, an aliquot of each was analyzed for sperm function, assessed as motility and forward progression, using CASA.

\subsection{EMF and Embryo Development}

To assess the effects of EMF on embryo development in culture, ten mice (strain CB6F1; Charles Rivers; Burlington, MA) were hyperstimulated using standard techniques. To prevent an animal bias, ten morphologically normal 2-cell embryos were collected from each mouse (100 embryos total) to be placed in culture. Embryos from a single mouse were then split randomly between control and EMF treatments. Embryos assigned to EMF treatment were exposed the $>200 \mathrm{mG}$ treatment above for $1 \mathrm{hr}$ and all embryos cultured for 5 days in Ham's F-10 media a 95\% relative humidity and $5 \% \mathrm{CO}_{2}$ (balance air). Embryos were assessed daily over a five day period for cellular development and normal appearance.

\subsection{Direct Exposure of Sperm Cells to Radio Frequency Radiation}

In addition to EMF exposure, gametes and early stage embryos in the ART laboratory might be subject to exposure to RF due to wireless equipment present in the laboratory. However, measurement of RF in the laboratory 
will vary with location and orientation to signaling equipment. To assess potential RF issues human sperm cells and early-stage mice embryos were subject to high levels of RF using a wireless router placed directly into the incubation equipment for maximum exposure.

Sperm samples from 5 paid donors were prepared for IUI using a standard sperm washing protocol. The final pellet was then resuspended in $1 \mathrm{~mL}$ of fresh Ham's F-10 and split into equal aliquots. One aliquot was incubated in the presence of the router for 24 hrs at $37 \mathrm{C}$, 95\% relative humidity and $5 \% \mathrm{CO}_{2}$ (balance air), the other aliquot was incubated similarly but without the router producing RF (control). Each sample was analyzed for motility and forward progression repeatedly over the 24 hrs using CASA.

\subsection{Statistical Analysis}

All data we analyzed by Chi Square, Student's t-test or ANOVA as appropriate. Analyses were conducted using the Statistical Package for the Social Sciences (SPSS; Chicago, IL). As expected, all motility parameters measured decreased with time $(\mathrm{P}<0.001)$. Therefore subsequence analyses were done within time point comparing treatments only to the control using paired t-test. Values were considered significant if $\mathrm{P}<0.05$.

\section{Results}

\subsection{Sperm Exposed to EMF}

Initial short-term exposure of porcine sperm cells to $>200 \mathrm{mG}$ of EMF proved to be detrimental to semen parameters. Both the motility $(\mathrm{P}<0.001)$ and forward progression $(\mathrm{P}<0.001)$ of cells exposed to the EMF decreased rapidly compared to the control at all time-points point past 0.5 hrs (Figure 1; motility only). Permeability studies with cells treated at the same EMF levels suggest these changes might have been induced by disruptive effects of the EMF on the cell membrane; as cells exposed to the field exhibited significantly higher rates of membrane permeability with $1 \mathrm{hr}$ of exposure to the EMF field (Figure 1).

Follow-up studies using the same porcine model but with exposure levels in the range seen from equipment in the lab (incubators, hoods and centrifuges) found no effect of the EMF until after 6 hrs and then only in those cells exposed to the highest ( $90 \mathrm{mG}$ ) levels of wave intensity. While levels consistent with the lower shelves of the incubator $(<30 \mathrm{mG})$ appeared to have no effect on motility $(\mathrm{P}=0.38)$ or forward progression $(\mathrm{P}=0.73)$, the higher levels decreased both motility and forward progression ( $\mathrm{P}<0.001$; Figure 2 -motility only).

However, studies with human sperm exhibit slightly different outcomes. While cell exposed to the EMF > $200 \mathrm{mG}$ demonstrated an average of 70\% loss of motility and lower forward progression scores 24 hrs after exposure $(\mathrm{P}<0.001)$, cells exposed to the lower levels of EMF maintained motilities $(\mathrm{P}=0.51)$ and forward progressions $(\mathrm{P}=0.89)$ similar to the control for the entire culture period.

\subsection{Embryos Exposed to EMF}

Results from embryos exposed to the high (>200 mG) concentrations of EMF were similar to those seen in

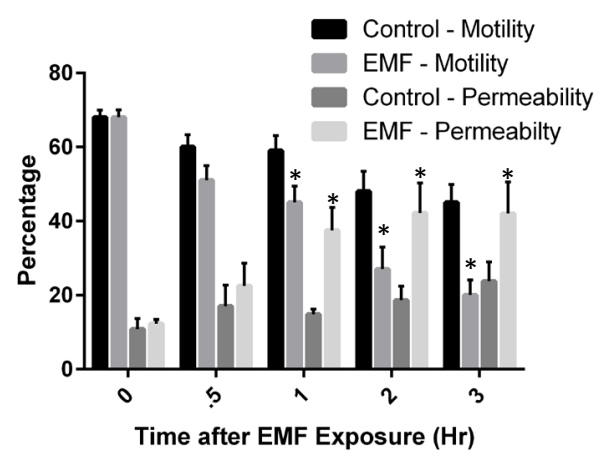

Figure 1. Influence of short-term exposure to high field strength (>200 mG) Electromagnetic Fields (EMF) in an assisted reproductive technology setting on porcine sperm motility and membrane permeability. EMF columns with asterisk are significantly different $(\mathrm{P}<$ 0.001) than controls at the same time point. 


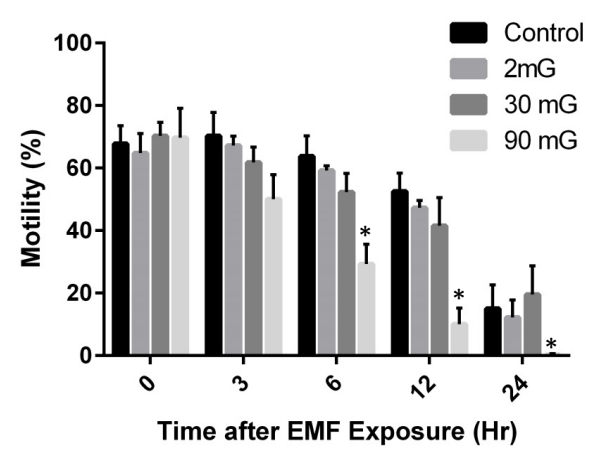

Figure 2. Influence of 24 hrs of exposure of varying field strengths of Electromagnetic Fields (EMF) on porcine sperm cell motility in an assisted reproductive technology setting on porcine sperm motility. EMF columns with asterisk are significantly different from controls at the same time point $(\mathrm{P}<0.001)$.

sperm. Mice embryos exposed to the EMF exhibited slower cell division $(\mathrm{P}<0.001)$ and more abnormal morphology $(\mathrm{P}<0.001)$ than embryos that were not exposed (control). This resulted in fewer embryos being at the proper developmental stage on day 3 - 5 of culture, meaning fewer of the embryos exposed to the EMF developed to blastocyst $(\mathrm{P}<0.001$; Figure 3$)$.

\subsection{Sperm Exposure to RF}

The most notable source of RF in the ART laboratory would be the wireless routers used for transmission of data. Field strength varies in direct proportion to distance from the device. Background readings in the laboratory ranged from 6 - $30 \mathrm{~W}$. Inside the incubator with the router off the measurement was $6 \mathrm{~W}$, while with the router on it measured approximately $300 \mathrm{~W}$ at the location sperm were placed to culture. Even at a 10X higher intensity that the highest level seen in background testing there were no apparent effects on either motility or forward progression ( $\mathrm{P}=0.65$; Figure 4-motility only).

\section{Discussion}

Various electrical fields and carrier waves generated by human activity have become a fact of everyday life. Considerable research has investigated the role of such sources of ionizing and non-ionizing radiation and their effects on human and animal health. Previous studies have demonstrated relationships between EMF and a variety of medical issues, including several types of cancers, neurological issues and other conditions [1] [2] [5] [7]. Further, a number of recent publications have raised concerns over the RF generated by various electronic devices and their potential roles in health issues [3] [4] [6]. Yet, both EMF and RF have also found beneficial rolls in medical diagnosis and treatment (NMR, ultrasound, ablation).

Both EMF and RF have been implicated in reproductive issues. Long-term exposures to EMF have been shown to decrease testicular function, sperm counts and motility in rats [7]. Other studies have suggested decreased motility and other issues in chronic users of electronic devices [11].

Given the highly technical nature of ART and the numerous pieces of electronic equipment present in the laboratory, there can be no doubt that both EMF and RF are present and to some extent interacting with gametes and embryos. However, to date little is known about the degree/magnitude of EMF, RF and potentially other carrier waves produced in the ART laboratory nor what potential risks they pose to normal fertilization and embryo development.

The present study examined the levels of both EMF and RF in an ART laboratory and applied equal levels in a controlled experimental setting. While EMF at the upper end of levels located within the laboratory were found to decrease sperm cell function and appeared to decrease embryo cell division, levels consistent with those measured at the actual location of gamete manipulation did not appear to effect cell group.

\section{Conclusion}

Electromagnetic fields and other carrier waves are present in the ART laboratory. However, while species diffe- 


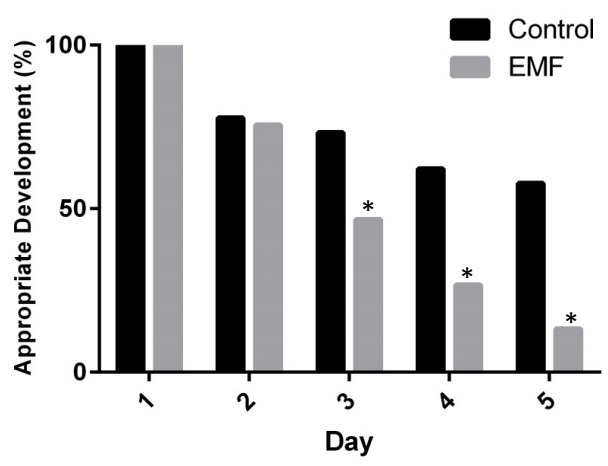

Figure 3. Influence of short-term (1 hr) exposure to high field strength (>200 mG) Electromagnetic Fields (EMF) on mice embryo development over 5 days of culture. EMF columns with asterisk are significantly different from controls at the same time point $(\mathrm{P}<$ 0.001).

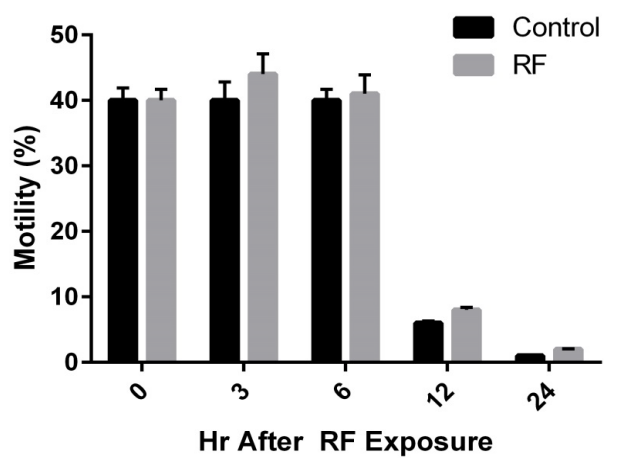

Figure 4. Twenty-four hrs of sperm exposure to high concentrations of cellphone-type radio frequency waves does not appear to influence human sperm cell motility $(\mathrm{P}=0.65)$.

rences appear to exist, under normal conditions, the intensity of these fields appears to have little effect on gamete and embryo function for the short duration the cells are exposed within the laboratory. It is recognized that the study did not examine all possible carrier waves present in the lab and that further study might be needed with EMF in the $80-100 \mathrm{mG}$ range, as these levels can occasionally be encountered by gametes and embryos in the lab (e.g. the upper shelve of conventional incubators) Given a recent study linking ART and congenital birth defects [12], further study might be warranted to assess long-term consequences of the exposure period. Further, the data suggests that laboratory personnel should be aware of the presence of such fields and take efforts to avoid overexposure of the gametes and embryos in their care.

\section{Acknowledgements}

The authors wish to acknowledge the technical support of Drs. Mathew Sellers, Lisa Welch and Carolina Praderio. We would also like to thank Ms. Leslie Alley for her assistance in editing the manuscript.

\section{References}

[1] Johansen, C. (2004) Electromagnetic Fields and Health Effects-Epidemiologic Studies of Cancer, Diseases of the Central Nervous System and Arrhythmia-Related Heart Disease. Scandinavian Journal of Work, Environment \& Health, 30, 1-30.

[2] Consales, C., Merla, C., Marino, C. and Benassi, B. (2102) Electromagnetic Fields, Oxidative Stress, and Neurodegeneration. International Journal of Cell Biology, 2012, Article ID: 683897.

[3] Coureau, G., Bouvier, G., Lebailly, P., Fabbro-Peray, P., Gruber, A., Leffondre, K., et al. (2014) Mobile Phone Use and Brain Tumours in the CERENAT Case-Control Study. Occupational and Environmental Medicine, 71, 514-522. 
http://dx.doi.org/10.1136/oemed-2013-101754

[4] Li, C.Y., Liu, C.C., Chang, Y.H., Chou, L.P. and Ko, M.C. (2012) A Population-Based Case-Control Study of Radiofrequency Exposure in Relation to Childhood Neoplasm. Science of the Total Environment, 1, 435-436, 472-478. http://dx.doi.org/10.1016/j.scitotenv.2012.06.078

[5] Kesari, K.K., Kumar, S., Nirala, J., Siddiqui, M.H. and Behari, J. (2013) Biophysical Evaluation of Radiofrequency Electromagnetic Field Effects on Male Reproductive Pattern. Cell Biochemistry and Biophysics, 65, 85-96. http://dx.doi.org/10.1007/s12013-012-9414-6

[6] Liu, K., Li, Y., Zhang, G., Liu, J., Cao, J., Ao, L. and Zhang, S. (2014) Association between Mobile Phone Use and Semen Quality: A Systemic Review and Meta-Analysis. Andrology, 2, 491-501. http://dx.doi.org/10.1111/j.2047-2927.2014.00205.X

[7] Hancı, H., Odacı, E., Kaya, H., Aliyazıcıŏlu, Y., Turan, İ., Demir, S., et al. (2013) The Effect of Prenatal Exposure to 900-MHz Electromagnetic Field on the 21-Old-Day Rat Testicle. Reproductive Toxicology, 42, 203-209.

[8] Lee, S.K., Park, S., Gimm, Y.M. and Kim, Y.W. (2014) Extremely Low Frequency Magnetic Fields Induce Spermatogenic Germ Cell Apoptosis: Possible Mechanism. BioMed Research International, 2014, Article ID: 567183. http://dx.doi.org/10.1155/2014/567183

[9] Rezk, A.Y., Abdulqawi, K., Mustafa, R.M., Abo El-Azm, T.M. and Al-Inany, H. (2008) Fetal and Neonatal Responses Following Maternal Exposure to Mobile Phones. Saudi Medical Journal, 29, 218-223.

[10] de Vocht, F. and Lee, B. (2014) Residential Proximity to Electromagnetic Field Sources and Birth Weight: Minimizing Residual Confounding Using Multiple Imputation and Propensity Score Matching. Environment International, 69, 5157.

[11] Mortazavi, S., Parsanezhad, M., Kazempour, M., Ghahramani, P., Mortazavi, A. and Davari, M. (2013) Male Reproductive Health under Threat: Short Term Exposure to Radiofrequency Radiations Emitted by Common Mobile Jammers. Journal of Human Reproductive Sciences, 6, 124-128. http://dx.doi.org/10.4103/0974-1208.117178

[12] Kelley-Quon, L.I., Tseng, C.H., Janzen, C. and Shew, S.B. (2013) Congenital Malformations Associated with Assisted Reproductive Technology: A California Statewide Analysis. Journal of Pediatric Surgery, 48, 1218-1224. http://dx.doi.org/10.1016/j.jpedsurg.2013.03.017 